\title{
Dedicated Psychiatry Clinic for Inflammatory Bowel Disease Patients Has a Positive Impact on Depression Scores
}

\author{
Kaleb Bogale ${ }^{a}$ Sanjay Yadav ${ }^{b}$ August Stuart ${ }^{c} \quad$ Allen R. Kunselman ${ }^{d}$ \\ Shannon Dalessio ${ }^{c}$ Nana Bernasko ${ }^{c}$ Andrew Tinsleyc Kofi Clarke ${ }^{c}$ \\ Emmanuelle Williams ${ }^{c}$ Matthew D. Coates ${ }^{c, e}$ \\ aPenn State University College of Medicine, Hershey, PA, USA; ${ }^{b}$ Department of Psychiatry, Penn State University \\ College of Medicine, Hershey, PA, USA; 'Division of Gastroenterology and Hepatology, Department of Medicine, \\ Penn State University College of Medicine, Hershey, PA, USA; dDepartment of Public Health Sciences, Penn State \\ University College of Medicine, Hershey, PA, USA; ${ }^{\text {} D e p a r t m e n t ~ o f ~ P h a r m a c o l o g y, ~ P e n n ~ S t a t e ~ U n i v e r s i t y ~ C o l l e g e ~ o f ~}$ \\ Medicine, Hershey, PA, USA
}

\section{Keywords}

Inflammatory bowel disease - Mental health - Psychological intervention · Crohn's disease · Ulcerative colitis

\begin{abstract}
Background: Psychiatric disorders, including anxiety and depression, are significantly more common in patients with inflammatory bowel disease (IBD). We established an integrated psychiatry clinic for IBD patients at our tertiary center IBD clinic to provide patients with critical, but frequently unavailable, coordinated mental health services. We undertook this study to evaluate the impact of this service on psychiatric outcomes, quality of life, and symptom experience. Methods: We performed a longitudinal prospective study comparing patients who had been cared for at our integrated IBD-psychiatry clinic to those who had not. We abstracted demographic and clinical information as well as contemporaneous responses to validated surveys. Results: Thirty-six patients cared for in the IBD psychiatry clinic were compared to a control cohort of 35 IBD patients. There was a significant reduction in the Hospital Anxiety and Depression Scale (HADS) depression score over time in the study cohort ( $p=$
\end{abstract}

karger@karger.com www.karger.com/iid

Karger!"

BOPEN ACCESS
(C) 2021 The Author(s)

Published by S. Karger AG, Basel

This is an Open Access article licensed under the Creative Common Attribution-NonCommercial-4.0 International License (CC BY-NC) (http://www.karger.com/Services/OpenAccessLicense), applicable to the online version of the article only. Usage and distribution for commercial purposes requires written permission.
0.001), though not in the HADS anxiety score. When compared to the control group, the study cohort showed a significant reduction in the HADS depression score. No significant differences were observed in the Harvey-Bradshaw Index, Simple Clinical Colitis Activity Index, or Short IBD Questionnaire. Conclusions: This is the first study to evaluate the impact of an integrated psychiatry clinic for IBD patients. Unlike their control counterparts, individuals treated in this clinic had a significant reduction in the mean HADS depression score. Larger scale studies are necessary to verify these findings. However, this study suggests that use of an integrated psychiatry IBD clinic model can result in improvement in mental health outcomes, even in the absence of significant changes in IBD activity. 02021 The Author(s).

Published by S. Karger AG, Basel

\section{Introduction}

Inflammatory bowel disease (IBD), including Crohn's disease (CD) and ulcerative colitis (UC), is a chronic disorder of the gastrointestinal tract that is associated with increased rates of psychiatric disorders, including anxiety 
and depression [1-3]. Comorbid anxiety and depression have each been shown to negatively impact patients with IBD as both have been associated with decreased treatment adherence [4-6], decreased quality of life [7-9], worsened perception of symptoms [10], and poorer disease course and outcomes [2,11-14]. Psychiatric interventions, including counseling, cognitive behavioral therapy, and hypnosis, have the potential to improve patient quality of life and to decrease rates of depression in IBD, particularly during periods of active disease [15-18]. As a result, provision of mental health services to IBD patients is becoming increasingly recommended in the literature, and there is an emerging effort to integrate mental health services to standard IBD treatment $[2,19,20]$. Unfortunately, these services are frequently unavailable, due to the relative limitation of access to mental health specialists (particularly those with experience working in the setting of IBD). Additionally, they may be avoided by patients due to the perceived social stigma of mental health treatment and/or the associated financial costs [21].

In an attempt to improve accessibility and timeliness of psychiatric care for IBD patients, we developed and integrated a dedicated psychiatry clinic within our IBD center. This clinic is undertaken on a weekly basis within our center and is run by a dedicated psychiatrist who is able to communicate and collaborate directly with the IBD providers, to discuss various aspects of patient care. Thus far, we have received very positive feedback from patients who have participated in this clinic. However, we were interested to determine if there were measurable differences in clinical outcomes as a result of this clinic. Thus, we undertook this pilot study to evaluate whether participation in this clinic resulted in significant differences in digestive disease and mental health-related outcomes in our patients.

\section{Methods}

\section{Study Design and Location}

We performed a prospective matched case-control study of patients cared for at the IBD Center at Pennsylvania State University Hershey Medical Center (PSHMC) between September 1, 2018, and August 30, 2020. The PSHMC is a tertiary care referral hospital in south-central Pennsylvania. This pilot study compared outcomes in cohorts of IBD patients who did and did not receive care in the integrated PSHMC IBD psychiatry clinic (IPC). All patients receiving integrated psychiatric care were seen by a psychiatrist (Dr. Sanjay Yadav [S.Y.]) who also oversaw this clinic. The IPC is located at the PSHMC IBD center as a collaborative initiative and supported by nursing staff specializing in management of digestive disease and dedicated IBD nurse navigators. Interventions under- taken in the IPC included an initial evaluation (with problem identification and diagnostic formulation), recommendations for appropriate behavioral activation, education on psychological responses to IBD illnesses, brief relaxation techniques, referral and resource identification for psychotherapy, referral to other integrated care services, and psychotropic medication initiation and management (where indicated). Please note that this study was performed in accordance with the internal rules and regulations of the Pennsylvania State University College of Medicine Institutional Review Board and carried out under protocol STUDY00013788.

\section{Inclusion and Exclusion Criteria}

Participants in this study were $>17$ years old and had an established diagnosis of CD, UC, or IBD colitis of an indeterminate nature, based upon standard clinical criteria routinely used to identify IBD. All study participants were evaluated at the IBD center and completed surveys relating to IBD activity (Harvey-Bradshaw Index [HBI], Simple Clinical Colitis Activity Index [SCCAI]), and quality of life (Short IBD Questionnaire [SIBDQ]) and mental health status (Hospital Anxiety and Depression Scale [HADS]) $[22,23]$ (see below) on at least 2 separate occasions, separated by a minimum of 3 months, during the study period.

Two cohorts were compared in this study: (1) IBD patients seen in the IBD center during the study period who had received psychiatric care defined as at least 1 visit to the IPC during the study period (the "study cohort") and (2) IBD patients, matched for age, disease subtype (CD vs. UC), sex, and baseline HADS anxiety and depression scores (see Table 1), who had been seen in the IBD center during the study period but had not received care in the IPC (the "control cohort"). Of note, the study cohort was identified first, and it included all of the patients who qualified for inclusion, using the criteria described above. The "control cohort" was consecutively selected from the remaining pool of patients receiving care at our IBD center, to match the size and characteristics of the "study cohort," by an individual who was blinded to all follow-up clinical scores and outcomes. Additionally, in the study cohort, the survey following care in the IPC was completed at least 3 months after that visit.

\section{Definitions and Data Abstraction}

The presence and relative severity of anxiety or depression symptoms were determined based upon responses to HADS surveys completed at the time of each clinical encounter. The HADS is a 14-item survey designed to specifically screen for these symptoms, using a series of simple questions that separately evaluate states of anxiety and depression [22]. The HADS has been validated to screen for anxious and depressed states in the setting of a variety of medical conditions, including IBD [23]. HADS scores for both anxiety and depression range from 0 to 7 (normal), 8-10 (borderline abnormal), and 11-21 (abnormal) [24]. Clinically significant anxiety or depression was defined as a HADS anxiety or HADS depression sub-score of 8 or more. Health-related quality of life was assessed using the SIBDQ [25]. The SIBDQ is a 10-question survey which scores on an inverse Likert scale, with 1 representing poor disease control and 7 representing optimal disease control, where the optimal health-related quality of life is a score of 70 and minimal health-related quality of life is a score of 10 . The HBI was used to assess for the severity of symptoms in CD patients [26]. The HBI score ranges from 0 to 4 (disease remission), 5-7 (mild disease), 8-16 (moderate disease), and $>16$ (severe disease).
Bogale et al. 
Table 1. Baseline characteristics of the control and study cohorts

\begin{tabular}{lllr}
\hline & $\begin{array}{l}\text { Control cohort } \\
(n=35)\end{array}$ & $\begin{array}{l}\text { Study cohort } \\
(n=36)\end{array}$ & $p$ value \\
\hline Age \pm SEM, years & $37.7 \pm 13.7$ & $39.0 \pm 10.0$ & 0.63 \\
Sex (female/male) & $25 / 10$ & $23 / 13$ & 0.62 \\
IBD type (CD/UC) & $28 / 7$ & $28 / 8$ & $>0.99$ \\
Initial HADS anxiety score & $9.4 \pm 0.5$ & $9.0 \pm 0.7$ & 0.68 \\
Initial HADS depression score & $5.2 \pm 0.5$ & $5.9 \pm 0.5$ & 0.38 \\
SIBDQ & $43.4 \pm 2.1$ & $44.2 \pm 2.0$ & 0.79 \\
HBI & $7.8 \pm 0.8$ & $7.1 \pm 0.9$ & 0.54 \\
SCCAI & $5.1 \pm 0.6$ & $4.7 \pm 0.5$ & 0.60 \\
\hline
\end{tabular}

SEM, standard error measurement; CD, Crohn's disease, UC, ulcerative colitis; HADS, Hospital Anxiety Depression Survey; SIBDQ, Short Inflammatory Bowel Disease Questionnaire; HBI, Harvey-Bradshaw Index; SCCAI, Short Clinical Colitis Activity Index.

Table 2. Longitudinal assessment of clinical outcomes

\begin{tabular}{|c|c|c|c|c|c|c|c|c|c|c|}
\hline & \multicolumn{4}{|c|}{ Control cohort $(n=35)$} & \multicolumn{4}{|c|}{ Study cohort $(n=36)$} & MDD $(95 \% \mathrm{Cl})$ & $p$ value \\
\hline HADS anxiety & 9.4 & 8.7 & $-0.7(-2.1,0.9)$ & 0.40 & 9.0 & 8.2 & $-0.8(-2.3,0.6)$ & 0.26 & $-0.1(-2.3,1.9)$ & 0.85 \\
\hline HADS depression & 5.2 & 5.1 & $-0.1(-1.1,0.8)$ & 0.81 & 5.9 & 4.3 & $-1.6(-2.5,-0.6)$ & 0.001 & $-1.4(-2.8,-0.1)$ & 0.03 \\
\hline SIBDQ & 43.4 & 46.1 & $+2.7(-1.3,6.8)$ & 0.19 & 44.2 & 47.2 & $3.0(-1.0,7.0)$ & 0.14 & $0.3(-5.4,6.0)$ & 0.92 \\
\hline
\end{tabular}

Bold numbers represent factors that were found to be significantly different during statistical comparison(s). The $p$ values reported above were determined by comparing paired changes from the initial visit to the follow-up visit in each in category between the control and study cohorts (using a 2-sample $t$ test). 95\% Cl, 95\% confidence interval; MDD, mean difference of differences; HADS, Hospital Anxiety Depression Survey; SIBDQ, Short Inflammatory Bowel Disease Questionnaire; HBI, Harvey-Bradshaw Index; SCCAI, Short Clinical Colitis Activity Index.

The SCCAI was used to assess for the severity of symptoms in UC patients [27]. The SCCAI ranges from 0 to 19, in which 0 represents no symptoms and 19 represents severe symptoms. Additional demographic and clinical characteristics were abstracted from the medical record, including patient age, sex, and disease subtype.

\section{Statistical Analysis}

The primary outcomes of interest were the HADS anxiety and depression scores, while secondary outcomes were the disease activity scores (HBI and SCCAI), and health-related quality of life (SIBDQ) (as defined above). Between cohort comparisons for demographic and clinical/psychiatric scores, variables were assessed using unpaired $t$ tests for continuous variables and $\chi^{2}$ tests (or Fisher's exact test if the expected cell count was $<5$ ) for categorical variables. Within cohort comparisons (i.e., difference between 2 time points per subject: [1] "baseline": at a clinic visit prior to psychiatric intervention and [2] "post-intervention": at a clinic visit at least 3 [3] months after intervention at the IPC) were assessed using paired $t$-tests for continuous variables. Parametric assumptions (e.g., normality of the distribution) for the $t$-tests were evaluated using residual diagnostics. All hypothesis tests were 2 sided, and analyses were performed using SAS software, version 9.4 (SAS Institute Inc., Cary, NC, USA), GraphPad Prism version 8 (San Di- ego, CA, USA), or R (R Core Team. R: A language and environment for statistical computing. R Foundation for Statistical Computing, Vienna, Austria. 2020. https:/www.R-project.org). Of note, $p$ values of $<0.05$ were considered to be statistically significant.

\section{Results}

\section{Description of Study Participants}

Utilizing the inclusion criteria described above, 36 patients were enrolled in the "study cohort" and 35 patients in the "control cohort." These cohorts had similar mean ages and baseline HADS, HBI, and SCCAI scores as well as similar distributions of gender and IBD types (Table 1).

Within the study cohort, the majority $(60 \%)$ were referred to the IPC for anxiety, while 32\% were referred for "distress" and 24\% for depression. Twenty (20) participants $(55.6 \%)$ had clinically significant symptoms of anxiety, while 11 (30.6\%) had clinically significant symptoms 
of depression. Thirteen individuals $(36.1 \%)$ were being treated with corticosteroids, and $8(22.2 \%)$ were treated with opiates. For the study cohort, the mean interval of time between visiting the IPC and the follow-up clinic visit was $9.0 \pm 0.8$ months (compared to $13.9 \pm 1.5$ months between the initial and follow-up clinic visits in the control cohort $[p=0.003])$.

\section{Impact of the Integrated IBD Psychiatry Clinic}

First, we evaluated HADS anxiety and depression scores over time in the study and control cohorts separately. In the study cohort, there was a significant decrease in the mean HADS depression score after the IPC intervention ( 5.9 vs. $4.3, p=0.001$ ) (Table 2). The number of patients with depression demonstrated a nonsignificant reduction (11 vs. $6, p=0.27$ ) after IPC intervention. No significant difference was observed in the mean HADS anxiety score ( 9.0 vs. $8.2, p=0.22$ ) or proportion of individuals with clinically significant symptoms of anxiety after being seen in the IPC ( 20 vs. $20, p=1.0$ ). When considering the clinical scores, while there was a reduction in the mean SCCAI ( 4.7 vs. 3.5, $p=0.04$ ), there were no significant differences in the HBI $(p=0.22)$ or SIBDQ (i.e., quality of life) scores over time (Table 2).

In the control cohort, no significant differences were found in the mean HADS anxiety (9.4 vs. 8.7, $p=0.40$ ) or HADS depression (5.2 vs. 5.1, $p=0.77$ ) scores or in the proportion of individuals with clinically significant scores of anxiety ( 26 vs. $20, p=0.21$ ) or depression (11 to $7, p=$ $0.41)$ over time. There were also no significant differences seen in the SIBDQ, HBI, or SCCAI scores over time.

Finally, we compared relative changes in the primary and secondary outcomes between the control and study cohorts. The psychiatric intervention cohort demonstrated a significantly greater drop in the mean HADS depression score than that in the control group $(p=0.03)$ (Table 2). There were no differences in the HADS anxiety score, SIBDQ, HBI, or SCCAI scores when comparing the control and study cohorts when considering the mean changes over time (Table 2).

\section{Discussion}

This is the first study to evaluate the impact of a dedicated and integrated psychiatric clinic for outpatient IBD patients. Unlike their control counterparts, patients treated in this clinic demonstrated significant improvements in depressive symptoms, even without measurable changes in disease severity scores or quality of life. Thus, this type of clinic appears to have a positive effect on depressive symptoms, even if IBD activity itself is not optimally controlled. Of note, no significant impact was exhibited in regard to symptoms of anxiety.

The results of this investigation support the findings of several previous investigations. It has previously been recognized that more formally integrating specialty clinics into the care of IBD patients can result in improvements in a variety of clinical outcomes. In regard to managing mental health of IBD patients specifically, 2 prior studies reported that screening patients with IBD for anxiety and/or depression and providing referrals to psychological care were effective in improving patient mental health (including depression) [20,28]. These studies also demonstrated that patients were more likely to pursue psychological treatment if it was directly associated with the referring provider group [20], highlighting the importance of incorporating an integrated model in this context. The promise of integrated care services may be best illustrated using the model of the IBD specialty medical "home," where specialty care services are coordinated for patients through a physician "champion" [29]. This approach to care has been associated with significant reductions in healthcare utilization, disease activity, and improvements in patient quality of life and psychiatric metrics [30].

Interestingly, the cohort receiving care in the IPC also demonstrated improvements in at least 1 disease activity score (e.g., SCCAI). Previous studies have demonstrated that increased levels of psychological stress are associated with more severe IBD activity [31] and may induce flares [32]. It is not clear whether stress management, psychotherapy, and/or other psychiatric interventions are helpful in managing luminal disease activity in the setting of IBD $[33,34]$, but studies such as ours hint at their potential in this context. Finally, as a result of our collaboration, it is worth noting that the psychiatrist overseeing the IPC (S.Y.) has developed significant familiarity and expertise in the care of IBD patients. It is quite possible that this experience has helped to improve his ability to provide specialized management of IBD patients and that individuals seen in the IPC feel more assured of receiving appropriate (and tailored) care.

There were several limitations to this study. First, this was a relatively small study, and the findings described above will need to be verified in larger cohorts of patients. As a result of the small size, it was impossible to effectively evaluate for certain potential confounders, including disease location, medication, and substance use or surgical history. There were also potentially useful types
Bogale et al. 
of data that were not collected in contemporaneous fashion, including endoscopically based disease activity assessments, laboratory values, and/or healthcare resource utilization. The mean interval follow-up time was also different between the control and study cohorts, and this may have influenced the outcomes. Additionally, we had a single psychiatrist providing care to patients. While we felt this might help reduce potential diagnostic and therapeutic biases that might exist among different providers, it is also possible that our results may be relatively unique to the work performed by our psychiatric care provider.

In spite of these limitations, our study evaluated data from very well-characterized cohorts. In addition, our findings demonstrated the efficacy of a dedicated clinic where patients can receive coordinated psychiatric and IBD care. Specifically, we showed that patients treated in this integrated psychiatry clinic had measurable improvements in depression, even without significant changes in measures of quality of life or certain measures of IBD activity severity. Further study of this intervention is certainly warranted. Future attempts to evaluate this topic should include larger cohorts of patients, in order to better investigate the potential role that medications, substance use, and a variety of disease-related factors have in this context. Nonetheless, this work already provides direct evidence for the value of integrating psychiatric specialty care in the context of IBD.

\section{Statement of Ethics}

Participants provided written consent to participate in this study. This study was approved by the Pennsylvania State University College of Medicine Institutional Review Board and carried out under protocol STUDY00013788.

\section{Conflict of Interest Statement}

The authors have no conflicts of interest to report in relation to this manuscript.

\section{Funding Sources}

This work was supported by the National Institutes of Health (NIH) National Institute of Diabetes and Digestive and Kidney Diseases (NIDDK) (R01 DK122364), the Peter \& Marsha Carlino Endowment in Inflammatory Bowel Disease (MDC), and the Margo E. Walrath Career Development Professorship in Gastroenterology (MDC).

\section{Author Contributions}

All the authors had full access to the data in the study, reviewed and edited the manuscript, and gave permission to submit the manuscript in its present form. Additionally, K.B. contributed to conceptualization, data abstraction, data analysis, and writing of the manuscript. S.Y. contributed to the project conceptualization, data analysis, and writing of the manuscript. S.D. contributed to formatting and editing the manuscript. A.K. and A.S. contributed to the data abstraction and analyses associated with the study. N.B., E.W., A.T., and K.C. contributed to data provision and conceptualization of the study as well as editing the manuscript. M.C. provided primary conceptualization, study design, interpretation of the data, manuscript development, and critical review.

\section{Data Availability Statement}

All data generated or analyzed during this study are included in this article. Further inquiries can be directed to the corresponding author.

\section{References}

1 Byrne G, Rosenfeld G, Leung Y, Qian $H$, Raudzus J, Nunez C, et al. Prevalence of anxiety and depression in patients with inflammatory bowel disease. Can J Gastroenterol Hepatol. 2017;2017:6496727.

2 Graff LA, Walker JR, Bernstein CN. Depression and anxiety in inflammatory bowel disease: a review of comorbidity and management. Inflamm Bowel Dis. 2009 Jul;15(7): $1105-18$.

3 Mikocka-Walus A, Knowles SR, Keefer L, Graff L. Controversies revisited: a systematic review of the comorbidity of depression and anxiety with inflammatory bowel diseases. Inflamm Bowel Dis. 2016 Mar;22(3):752-62.
4 Nigro G, Angelini G, Grosso SB, Caula G, Sategna-Guidetti C. Psychiatric predictors of noncompliance in inflammatory bowel disease: Psychiatry and compliance. J Clin Gastroenterol. 2001 Jan;32(1):66-8.

5 Shale MJ, Riley SA. Studies of compliance with delayed-release mesalazine therapy in patients with inflammatory bowel disease. $\mathrm{Al}$ iment Pharmacol Ther. 2003 Jul 15;18(2): 191-8.

6 Nahon S, Lahmek P, Durance C, Olympie A, Lesgourgues B, Colombel JF, et al. Risk factors of anxiety and depression in inflammatory bowel disease. Inflamm Bowel Dis. 2012 Nov; 18(11):2086-91.
7 Zhang CK, Hewett J, Hemming J, Grant T, Zhao $\mathrm{H}$, Abraham C, et al. The influence of depression on quality of life in patients with inflammatory bowel disease. Inflamm Bowel Dis. 2013 Jul;19(8):1732-9.

8 Guthrie E, Jackson J, Shaffer J, Thompson D, Tomenson B, Creed F. Psychological disorder and severity of inflammatory bowel disease predict health-related quality of life in ulcerative colitis and Crohn's disease. Am J Gastroenterol. 2002 Aug;97(8):1994-9.

9 Hjortswang H, Jarnerot G, Curman B, Sandberg-Gertzen $\mathrm{H}$, Tysk $\mathrm{C}$, Blomberg B, et al. The influence of demographic and diseaserelated factors on health-related quality of life in patients with ulcerative colitis. Eur J Gastroenterol Hepatol. 2003 Sep;15(9):1011-20. 
10 Navabi S, Gorrepati VS, Yadav S, Chintanaboina J, Maher S, Demuth P, et al. Influences and impact of anxiety and depression in the setting of inflammatory bowel disease. Inflamm Bowel Dis. 2018 Oct 12;24(11):2303-8.

11 Maunder RG. Evidence that stress contributes to inflammatory bowel disease: evaluation, synthesis, and future directions. Inflamm Bowel Dis. 2005 Jun;11(6):600-8.

12 Maunder RG, Levenstein S. The role of stress in the development and clinical course of inflammatory bowel disease: epidemiological evidence. Curr Mol Med. 2008 Jun;8(4):24752.

13 Mittermaier C, Dejaco C, Waldhoer T, Oefferlbauer-Ernst A, Miehsler W, Beier M, et al. Impact of depressive mood on relapse in patients with inflammatory bowel disease: a prospective 18-month follow-up study. Psychosom Med. 2004 Jan;66(1):79-84.

14 Narula N, Pinto-Sanchez MI, Calo NC, Ford AC, Bercik P, Reinisch W, et al. Anxiety but not depression predicts poor outcomes in inflammatory bowel disease. Inflamm Bowel Dis. 2019 Jun 18;25(7):1255-61.

15 Paulides E, Boukema I, van der Woude CJ, de Boer NKH. The effect of psychotherapy on quality of life in ibd patients: a systematic review. Inflamm Bowel Dis. 2021 Apr 15;27(5): 711-24.

16 Bennebroek Evertsz F, Sprangers MAG, Sitnikova K, Stokkers PCF, Ponsioen CY, Bartelsman J, et al. Effectiveness of cognitive-behavioral therapy on quality of life, anxiety, and depressive symptoms among patients with inflammatory bowel disease: a multicenter randomized controlled trial. J Consult Clin Psychol. 2017 Sep;85(9):918-25.

17 Wynne B, McHugh L, Gao W, Keegan D, Byrne K, Rowan C, et al. Acceptance and commitment therapy reduces psychological stress in patients with inflammatory bowel diseases. Gastroenterology. 2019 Mar;156(4):935-45. e1.
18 Gracie DJ, Irvine AJ, Sood R, Mikocka-Walus A, Hamlin PJ, Ford AC. Effect of psychological therapy on disease activity, psychological comorbidity, and quality of life in inflammatory bowel disease: a systematic review and meta-analysis. Lancet Gastroenterol Hepatol. 2017 Mar;2(3):189-99.

19 Bannaga AS, Selinger CP. Inflammatory bowel disease and anxiety: Links, risks, and challenges faced. Clin Exp Gastroenterol. 2015;8: 111-7.

20 Lores T, Goess C, Mikocka-Walus A, Collins KL, Burke ALJ, Chur-Hansen A, et al. Integrated psychological care is needed, welcomed and effective in ambulatory inflammatory bowel disease management: evaluation of a new initiative. J Crohns Colitis. 2019 Jul 25;13(7):819-27.

21 Taft TH, Ballou S, Bedell A, Lincenberg D. Psychological considerations and interventions in inflammatory bowel disease patient care. Gastroenterol Clin North Am. 2017 Dec; 46(4):847-58.

22 Zigmond AS, Snaith RP. The hospital anxiety and depression scale. Acta Psychiatr Scand. 1983 Jun;67(6):361-70.

23 Yamamoto-Furusho JK, Sarmiento-Aguilar A, Garcia-Alanis M, Gomez-Garcia LE, Toledo-Maurino J, Olivares-Guzman L, et al. Hospital anxiety and depression scale (hads): validation in mexican patients with inflammatory bowel disease. Gastroenterol Hepatol. 2018 Oct;41(8):477-82.

24 Stern AF. The hospital anxiety and depression scale. Occup Med. 2014 Jul;64(5):393-4.

25 Irvine EJ, Zhou Q, Thompson AK. The short inflammatory bowel disease questionnaire: a quality of life instrument for community physicians managing inflammatory bowel disease. Ccrpt investigators. Canadian Crohn's relapse prevention trial. Am J Gastroenterol. 1996 Aug;91(8):1571-8.

26 Harvey RF, Bradshaw JM. A simple index of Crohn's-disease activity. Lancet. 1980 Mar 8; 1(8167):514.
27 Walmsley RS, Ayres RC, Pounder RE, Allan RN. A simple clinical colitis activity index. Gut. 1998;43(1):29-32.

28 Lores T, Goess C, Mikocka-Walus A, Collins KL, Burke ALJ, Chur-Hansen A, et al. Integrated psychological care reduces health care costs at a hospital-based inflammatory bowel disease service. Clin Gastroenterol Hepatol. 2021 Jan;19(1):96-103.e3.

29 Regueiro MD, McAnallen SE, Greer JB, Perkins SE, Ramalingam S, Szigethy E. The inflammatory bowel disease specialty medical home: a new model of patient-centered care. Inflamm Bowel Dis. 2016 Aug;22(8):1971-80.

30 Regueiro M, Click B, Anderson A, Shrank W, Kogan J, McAnallen S, et al. Reduced unplanned care and disease activity and increased quality of life after patient enrollment in an inflammatory bowel disease medical home. Clin Gastroenterol Hepatol. 2018 Nov; 16(11):1777-85.

31 Araki M, Shinzaki S, Yamada T, Arimitsu S, Komori M, Shibukawa N, et al. Psychologic stress and disease activity in patients with inflammatory bowel disease: a multicenter cross-sectional study. PLoS One. 2020;15(5): e0233365.

32 Sun Y, Li L, Xie R, Wang B, Jiang K, Cao H. Stress triggers flare of inflammatory bowel disease in children and adults. Front Pediatr. 2019;7:432.

33 Boye B, Lundin KE, Jantschek G, Leganger S, Mokleby $\mathrm{K}$, Tangen $\mathrm{T}$, et al. Inspire study: does stress management improve the course of inflammatory bowel disease and diseasespecific quality of life in distressed patients with ulcerative colitis or Crohn's disease? A randomized controlled trial. Inflamm Bowel Dis. 2011 Sep;17(9):1863-73.

34 McCombie AM, Mulder RT, Gearry RB. Psychotherapy for inflammatory bowel disease: a review and update. J Crohns Colitis. 2013 Dec;7(12):935-49. 